\title{
Cystic lymphangioma of breast due to filarial infestation: a rare entity
}

\begin{abstract}
Cystic lymphangiomas are a congenital malformation of the lymphatic system resulting from sequestration of the lymphatic tissue, which no longer interacts normally with the surrounding lymphatic system.1 Lymphangioma commonly occur in the neck $(70 \%)$, axilla $(30 \%)$ or abdomen $(10 \%) 2$ and are rare in the breast. A $45 y$ year old female presented in the surgery outpatient department with complains of increasing pain and heaviness in the right breast for 4months with gradual increase in size of the same breast with no history of nipple discharge and no history suggestive trauma. On examination, a $15 \times 18 \mathrm{~cm}$ diffuse swelling was present in the right breast involving all the quadrants and extending into right axilla and chest wall upto the clavicle. Swelling was soft with smooth surface, compressible and transilluminant with no axillary lymph nodes. Mammography showed high density lesion suggestive of fibroadenosis. Ultrasonography showed multiple cysts of different sizes and shapes with sepatations, largest cyst measured $2.9 \times 1.4 \mathrm{~cm}$. MRI was planned to evaluate the extent of the lesion which revealed right breast lymphatic malformations with subcutaneous and cutaneous mammary congestion on right, multiple non mass enhancing areas in right breast extending into the right axilla and right chest wall upto the clavicle. FNAC was performed under USG guidance cytology revealed lymphocytic preponderance which suggested a benign cystic lesion. The lymphatic malformation involving the right breast was excised through a periareolar incisiona and a separate incision given in the axilla. Histopathology of the specimen was suggestive of cystic lymphangiom as with filarial infiltration into the lymph nodes.USG guided intralesional Bleomycin injection was given in the remnant supraclavicular region. Patient did well during the post operative course.
\end{abstract}

Volume 4 Issue $6-2017$

\author{
Seema Khanna \\ Department of General Surgery, Banaras Hindu University, India
}

Correspondence: Seema Khanna, Department of General Surgery, Institute of Medical Sciences, Banaras Hindu University, Varanasi, 22I 005, India, Tel 0542-23I7678,

Email seemakhannal19@rediffmail.com

Received: January 31, 2017| Published: April 17, 2017

\section{Background}

Cystic lymphangiomas are a congenital malformation of the lymphatic system resulting from sequestration of the lymphatic tissue, which no longer interacts normally with the surrounding lymphatic system. ${ }^{1}$ More than $50 \%$ of these lesions are present at birth, $90 \%$ before the age of 2years. Most of the cystic lymphangioma occur in the neck $(70 \%)$ or axilla $(30 \%)$ followed by abdomen $(10 \%){ }^{2}$ Cystic lymphangiomas of the breast are extremely rare. They may be congenital malformations or acquired (secondary to trauma, infection or neoplasia) in the mammary gland. These lesions tend to infiltrate surrounding tissues and malignant degeneration is extremely rare. This case report concerning a 45year female with a rare cystic lymphangioma of the breast gave us the possibility to critically evaluate the diagnosis and treatment of this lesion.

\section{Procedure}

A 45-year-old female presented in the surgery outpatient department with complains of increasing pain and heaviness in the right breast for 4 months along with a gradual increase in size of the same breast. There was no history of nipple discharge or rapid increase in size of the swelling. There was no history suggestive of fever, trauma or any other swelling in the body. Family history was not contributary. On examination, left breast was normal. A $15 \times 18 \mathrm{~cm}$ diffuse swelling was present in the right breast involving all the quadrants and extending into right axilla and chest wall upto the clavicle. Swelling was soft with smooth surface, compressible and transilluminant. No axillary lymph nodes were palpable. Mammography showed high density lesion suggestive of fibroadenosis. High resolution USG was performed which showed multiple cysts of different sizes and shapes with separations within the cyst, largest cyst measured $2.9 \times 1.4 \mathrm{~cm}$. MRI was planned to evaluate the extent of the lesion which revealed right breast lymphatic malformations with subcutaneous and cutaneous mammary congestion on right, multiple non mass enhancing areas in right breast extending into the right axilla and right chest wall upto the clavicle. The cyst spaces appeared hyperintense on both $\mathrm{T} 1$ and $\mathrm{T} 2$ weighted fat suppressed images, whereas the septae appeared hypointense. FNAC was performed under USG guidance and $10 \mathrm{ml}$ of yellowish fluid was aspirated. Cytology revealed lymphocytic preponderance which suggested a benign cystic lesion. The lymphatic malformation involving the right breast was excised through a periareolar incision. A separate incision was given in the axilla and the axillary mass was excised upto the axillary vein. The axillary mass showed enlarged axillary lymph nodes and multiple cystic swelling extending into the neck, over the axillary artery and brachial plexus. Histopathology of the specimen revealed multiple cystic lesions suggestive of Cystic lymphangiomas with filarial infiltration into the lymph nodes. Patient did well during the postoperative period. USG guided intralesional Bleomycin injection was given in the remnant supraclavicular region swelling. While keeping the tip of aspiration needle within a cyst lumen, $0.5 \mathrm{mg}$ per $\mathrm{kg}$ body weight of bleomycin aqueous solution $(1.5 \mathrm{mg} / \mathrm{ml}$ water) was injected. The calculated dose was divided by the number of cysts aspirated and the divided dose was injected into each cyst .This procedure was repeated after 4 weeks as the cystic component persisted for three cycles.

\section{Discussion}

Filariasis is endemic in large areas of India, Africa, and Far East. 
Wuchereria bancrofti (W. bancrofti) accounts for approximately $90 \%$ of all filariasis cases in the world followed by Brugia malayi (B. malayi) and Brugia timori (B. timori). W.bancrofti is predominant species. It has not been reported from areas endemic for B.malayi. The larvae enter the lymphatic vessels causing lymphangitis. When the female breast is involved, the larvae enter the lymphatic vessels causing lymphangitis, fibrosis and disruption of lymphatic drainage. ${ }^{3}$ Hyperemia in the overlying skin with changes of peau d' orange and enlargement of axillary lymph nodes has also been reported which makes it important to rule out carcinoma. Demonstration and identification of the parasite in the smear plays a significant role in the prompt recognition of the disease and institution of specific therapy. Histopathology usually can confirm the diagnosis by finding of an eosinophilic granulomatous reaction around the filarial parasites which are in varying stages of degeneration ${ }^{4}$ Lymphangiomas are lympho-venous malformations and are believed to be sequestered off from lymphatic spaces that fail to establish connections with the main lymphatic channels. ${ }^{5,6}$ Cystic lymphangioma of the breast in an adult woman was first described by Sieber et al., ${ }^{7} 90 \%$ of these occur in the neck or axilla ${ }^{1,2}$ and the remaining $10 \%$ in abdominal organs, the retroperitoneum, skeleton, scrotum and, very rarely, the breast. ${ }^{1,8}$ Cystic lymphangiomas of the breast are very rare. Some authors regard lymphangiomas to be true neoplasms that are capable of local aggressive behaviour, but overall they are benign. ${ }^{9}$ They may be congenital or secondary to other etiologic factors of cystic lymphangioma including obstruction of the lymphatic channels with secondary dilatation, congenital weakness of the lymphatic wall and proliferation of the lymphatic vessels ${ }^{10}$ which explains our case of filarial infiltration into the axillary lymphatics leading to cystic lymphangioma of the breast lymphatics. Cystic lymphagioma may also be associated with chromosomal abnormalities and other anatomical anomalies including Turner's syndrome, trisomy 21 and trisomy 18.

Lymphangiomas have been classified into the simple, cavernous and cystic types. Simple lymphangioma consists of several small, capillarysized vessels; cavernous lymphangioma, is composed of dilated lymphatic vessels surrounded by a fibrous capsule and a lymphatic stroma containing lymphoid aggregates. Cystic lymphangioma is characterized by large, cyst-like spaces filled with clear lymph fluid, lined by endothelial cells, with well-defined margins; it is not encapsulated. ${ }^{11}$ Lymph vessels of the adult mammary gland originate in the interlobular connective tissue and the walls of the lactiferous ducts. These communicate with the overlying cutaneous lymphatic plexus, especially around the nipple in the subareolar plexus, and then drain to the axilla. ${ }^{6}$ Lymphangiomas may be diffuse or multicentric (lymphangiomatosis). They may involve only soft tissues or may be associated with bone and/or visceral lesions. ${ }^{12}$ A lymphangioma is usually a smooth, asymptomatic mass; Complications include infection, hemorrhage, and fistula formation. Rapid expansion may occur, due to hemorrhage into the cyst, inflammation or trauma. These lesions enlarge over time by the collection of fluid. Physical examination reveals a well-circumscribed, non tender, smooth and mobile mass on palpation. ${ }^{10,12}$ Localized cystic lymphangiomas of the breast can occur in the upper outer quadrant, the tail of Spence or in the subareolar space. In our case, the entire breast was affected. The differential diagnosis include simple cysts, hematoma, lymphocele, and hemangioma. Simple breast cysts are usually bilateral and often occur in other regions of the breast. Hematomas are localized, unilocular. Hemangiomas show intense enhancement after gadolinium administration, but some may even show gradual filling in of contrast on delayed scans up to $30 \mathrm{~min}$ later.

Breast cystic lymphangiomas show round or lobulated densities on mammography with no macro or micro-calcifications. ${ }^{13}$ On ultrasonography, cystic hygroma appears as a radiopaque, multiloculated, cystic mass with linear septa of variable thickness that contain solid elements originating from the cyst walls or septa. ${ }^{14}$ Larger lesions may not have well defined margins and cysts may extend to surrounding tissues. ${ }^{15}$ In controversial cases, computed tomography (CT) and magnetic resonance imaging (MRI) can be useful. CT provides an accurate image of the lesion after intravenous injection of contrast medium for enhancement of the lymph vessels. MRI allows better typification of the lesion because it provides a different multiplanar resolution and better detail of surrounding soft tissues, the depth and extent of the tumour thereby helping therapy. ${ }^{16}$ The cystic spaces are usually filled with clear fluid that appears hypointense on $\mathrm{T} 1$ and hyperintense on T2-weighted imaging. The fluid at times appears hyperintense on both $\mathrm{T} 1$ and $\mathrm{T} 2$-weighted sequences, suggesting the presence of hemorrhage or increased protein levels which may indicate inflammation. ${ }^{8}$ Since radiographic images are not always sufficient to make a definite diagnosis, fine-needle aspiration biopsy is often performed although it is not always diagnostic,it can be useful in the excluding certain diagnosis.Cytological examination shows a clear or yellowish hypocellular fluid containing a variable number of mature lymphocytes and no atypical cells. ${ }^{16,17}$

Cystic lymphangioma does not usually degenerate into a malignant tumor; surgical excision is the treatment of choice for this kind of lesion. Failure to treat may result in secondary complications such as infection and haemorrhage. The greatest difficulty in treatment is related to the size and location of the mass. It may be difficult to obtain safe margins due to the tendency of these lesions to infiltrate surrounding tissues as in our case; there is a high risk of incomplete excision, possibly leading to rapid recurrence. ${ }^{18}$ Sclerotherapy, incision, drainage, irradiation, and cryotherapy have proved to be ineffective and may carry risks such as hemorrhage and infection. ${ }^{19,20}$ After incomplete surgical excision, recurrences may develop but if complete surgical excision is attempted it can cause aesthetic or nerve damage when an extensive surgical dissection is necessary to remove large lesions. ${ }^{21}$ In such cases the other treatment options such as intralesional sodium tetra decylsulphate, bleomycin or OK432 can be resorted to under USG guidance. Intralesional sclerotherapy has become an acceptable method of treatment for lymphangiomas. It involves the use of a sclerosing agent that causes irritation of the endothelial lining of the lymphangioma, which leads to inflammation, fibrosis and involution. Different authors have quoted success rates of between 36 to $63 \%$ for complete tumour regression, of up to $88 \%$ significant lesion regression, and poor response of between 12 to $23 \%$ using bleomycin. ${ }^{20-23}$ Patient should be councelled regarding recurrence and should be kept on monthly follow up under USG.

\section{Conclusion}

Cystic lymphangioma is a rare congenital malformation of the lymphatic system characterized by the presence of non-communicating lymphatic channels, resulting in the formation of multiple cystic spaces. Although their prevalence is very rare, cystic lymphangioma should be considered in the differential diagnosis of an irregular cystic mass of the breast. They can be diagnosed from their characteristic imaging findings and typical locations, such as the subareolar area 
and outer upper quadrant of the breast. The diagnosis can be also suggested by fine-needle aspiration cytologic findings. Though MR imaging may be expensive and not freely available, it is very helpful in characterizing the lesion and identifying its extent, because of its multiplanar imaging ability.

\section{Acknowledgements}

None.

\section{Conflict of interest}

The author declares no conflict of interest.

\section{References}

1. Enzinger FM, Weiss SW. Tumors of Lymph Vessels in Soft Tissue Tumours. 3rd ed. USA: Mosby Year Book, Inc; 1995. p. 679-688.

2. Emery PJ, Bailey CM, Evans JNG. Cystic hygroma of the head and neck. J Laryngol Otol. 1984;98(6):613-619.

3. Alkadhi H, Garzoli E. Calcified filariasis of the breasts. $N$ Engl J Med. 2005;352(2):e2.

4. Dayal A, Selvaraju K, Dayal A. Filariasis of the breast. Webmed Central Surgery. 2008;1(11):WMC00942.

5. Menville JG, Bloodgood JC. Subcutaneous angio mas of breast. Ann Surg. 1933;97(3):401-413.

6. Kangesu T. Cystic hygroma of the breast in childhood. Br J Clin Pract. 1990;44:787-788.

7. Sieber PR, Sharkey FE. Cystic hygroma of the breast. Arch Pathol Lab Med. 1986;110(4):353.

8. Torcasio A, Veneroso S, Amabile MI, et al. Cystic hygroma of the breast: a rare lesion. Tumori. 2006;92(4):347-350.

9. Weiss SW, Goldblum JR. Enzinger and Weiss's Soft tissue tumours. USA Tumour of Lymph vessels; 2001. p. 955-958.

10. Sa EJ, Choi YH. Cystic lymphangioma of the breast. J Clin Ultrasound. 1999;27(6):351-352.
11. Kumar V, Abbas AK, Fausto N. Robbins \& Cotran Pathologic Basis of Disease. 7th ed. UK: Saunders; 2005. 547 p.

12. Ramani P, Shah A. Lymphangiomatosis. Histologic and immunohistochemical analysis of four cases. Am J Surg Pathol. 1993;17:329 335 .

13. Kook SH, Keum JS. Mammography, US and MR findings of cystic lymphangioma of the breast: a case report. J Korean Radiol Soc. 1996;35(2):279-281.

14. Chung SY, Oh KK, Kim DJ. Mammographic and sonographic findings of a breast cystic lymphangioma. J Ultrasound Med. 2003;22(3):307-309.

15. Sheth S, Nussbaum AR, Hutchins GM, et al. Cystic hygromas in children: sonographic-pathologic correlation. Radiology. 1987;162(3):821-824.

16. Sarno RC, Carter BL, Bankoff MS. Cystic lymphangiomas: CT diagnosis and thin needle aspiration. Br J Radiol. 1987;57(677):424-426.

17. Henke AC, Cooley ML, Hughes JH, et al. Fine needle aspiration cytology of lymphangioma of the parotid glad in an adult. Diagn Cytopathol. 2001;24(2):126-128.

18. Okada A, Kubota A, Fukuzawa M, et al. Injection of bleomycin as a primary therapy of cystic lymphangioma. J Pediatr Surg. 1992;27(4):440 443.

19. Kwon SS, Kim SJ, Kim L, et al. Huge cystic lymphangioma involving the entire breast. Ann Plast Surg. 2009;62(1):18-21.

20. Zulfiqar MA, Zaleha AM, Zakaria Z. The treatment of neck lymphangioma with intralesional injection of bleomycin. Med JMalaysia. 1999;54(4):478 481 .

21. Tanigawa N, Shimomatsuya T, Takahashi K, et al. Treatment of cystic hygroma and lymphangioma with the use of bleomycin fat emulsion. Cancer. 1987;60(4):741-749.

22. Mahajan JK, Bharathi V, Chowdhary SK, et al. Bleomycin as intralesional sclerosant for cystic hygromas. J Indian Assoc Pediatr Surg. 2004;9(1):3-

23. Molitch HI, Unger EC, Witte CL, et al. Percutaneous sclerotherapy of lymphangiomas. Radiology. 1995;194(2):343-347. 